\title{
Radiated Noise Measurement of Ships Based on Stochastic Resonance
}

\author{
Chao Peng ${ }^{1}$, HaiXin Sun $^{2}$ and En Cheng ${ }^{*}, 1$ \\ ${ }^{I}$ Key Laboratory of Underwater Acoustic Communication and Marine Information Technology Ministry of Education, \\ Xiamen University, Xiamen, Fujian, 361005, China \\ ${ }^{2}$ School of Electronic Engineering, Dongguan University of Technology, Dongguan, Guangdong, 523808, China
}

\begin{abstract}
The radiated noise of ships involves strong low-frequency line spectrum, which is greatly related with the working states of auxiliary equipment and the rotation of propeller, and then regarded as the important basis in feature extraction of ships. Moreover, the stochastic resonance has special advantages in the extraction of weak signal. Aiming at the multi-frequency components in radiated noise of the ship with four-blade propeller, and the characteristic without adiabatic approximation assumption, a sweep-frequency stochastic resonance detection system is established in this paper. Meanwhile, a kind of detection method on multi-frequency signal with partitioning large parameters is proposed, in order to identify the components of low-frequency line spectrum in radiated noise of ship. The analysis and simulation results present that, under the strong noise background, the proposed method can still effectively detect the low-frequency line spectrum, which lays the solid foundation for the subsequent target recognition and speed estimation of the ship.
\end{abstract}

Keywords: Low-frequency line spectrum detection, multi-frequency signal with large parameters, ship radiated noise, stochastic resonance.

\section{INTRODUCTION}

Through the researches on generation mechanism of ship radiated noise [1], its source can be divided into three types, including mechanical noise, propeller noise and hydrodynamic noise. Concretely speaking, the hydrodynamic noise belongs to irregular noise. The mechanical noise, generated from the mechanical vibration of working units in ship, is the main component of ship radiated noise in the condition of low ship speed. Meanwhile, the propeller noise, generated from the blade resonance when the propeller blade flaps and cuts the water flow, belongs to a type of mixed noise radiated by propeller, and its main component is "singing" [2]. Moreover, the propeller noise is the main component of ship radiated noise in low-frequency band 1$100 \mathrm{~Hz}$. It is investigated that [3], when the cavitation appears during the enhancement of ship speed, the line spectrum related to propulsion system moves towards highfrequency zone, and its magnitude increases. However, the line spectrum of auxiliary equipment is basically unchanged, and this low-frequency line spectrum is an important basis for target recognition and speed estimation of the ship.

In 1981, the idea of stochastic resonance was proposed by the physicist Benzi in Italy and the physicist Suterz in USA, et al. [4], in order to explain the periodicity of earth glacier. Stochastic resonance, produced by nonlinear system, stochastic force (noise) and input signal, is a peculiar synergetic phenomenon, which reflects the positive role of the stochastic force.

*Address correspondence to this author at the Key Laboratory of Underwater Acoustic Communication and Marine Information Technology Ministry of Education, Xiamen University, Xiamen, Fujian, 361005, China; E-mail: chengen@xmu.edu.cn
Hu Gang et al. proposed the adiabatic approximation theory [5] and proved that the stochastic resonance effect was suitable for detecting the small parameters signal. However, in the low-frequency line spectrum of ship radiated noise, it always contains multi-frequency components, most of which do not meet the adiabatic approximation assumption, i.e., the signal frequency is far less than $1 \mathrm{~Hz}$. Therefore, the traditional stochastic resonance system cannot meet the requirements any more. As a result, we put forward a sweep-frequency stochastic resonance detection system in this paper. In first, we analyze the low-frequency line spectrum signal, and estimate the frequency range of the signal to be measured. Then, in this range, we carry out partitioning adjustment on the frequency of carrier sweeping signal, which guarantees that the frequency mixing signal can satisfy the adiabatic approximation assumption in order that the stochastic resonance is generated. Subsequently, we estimate the power spectrum of the output signal, and detect the low-frequency line spectrum signal.

\section{THEORETICAL ANALYSIS}

\subsection{Bistable System and Its Performance Analysis}

Stochastic resonance is a typical nonlinear system, and its classical model is the following bistable system [6]:

$\frac{d x}{d t}=a x-b x^{3}+A \cos (2 \pi f \mathrm{t})+n(\mathrm{t})$

In Eq. (1), $a$ and $b$ are the system parameters of bistable system, $A \cos (2 \pi f \mathrm{t})$ is the weak signal to be measured, where $A$ is the magnitude of signal, and $f$ is the frequency of signal. Here, the weak signal is not only 
drowned in the noise signal in time domain, but also presents no obvious signal spectrum peak to distinguish signal and noise in frequency domain. $n(\mathrm{t})$ is the Gauss white noise, whose mean value is $E[n(\mathrm{t})]=0$ and autocorrelation function is $E[n(\mathrm{t}) \mathrm{n}(\mathrm{t}-\tau)]=2 D \delta(\tau)$. Here, $D$ is the noise intensity, and the noise variance is equal to $2 D$.

We usually use the output signal-to-noise ratio (SNR) as an index to measure the degree of resonance [7], where SNR is defined as the ratio of signal power at the measured frequency point and the remainder total power except for this frequency point:

$S N R_{\text {out }}=10 \times \log _{10}\left(\frac{P_{\text {signal }}}{P_{\text {noise }}}\right)$

where, $P_{n o i s e}$ is the noise power, and it is obtained by calculating the average of noise powers in a certain scope of corresponding signal frequency. However, because of the randomness of stochastic resonance system, it is not tenable to use the output SNR as a index to measure the degree of resonance. Subsequently, in this paper, we use the SNR gain as the index to measure the strength of stochastic resonance:

$S N R_{\text {gain }}=\frac{S N R_{\text {out }}}{S N R_{\text {in }}}$

Adjusting the noise intensity $D$ or system parameters can both make the SNR gain reach a maximum value, and the effect of these two methods are identical [8]. However, these two regulation methods have both advantages and disadvantages. In concrete, parameter adjustment involves the two system parameters with mutual influence, and there is no explicit mathematical expression on the relationship of them. Moreover, the regulation of noise is very difficult in practical engineering, and being continued to increase the noise becomes meaningless when the noise intensity exceeds the cooperative scope of system and signal. As a result, in order to reach the best state of stochastic resonance system, system parameters and noise must be simultaneously adjusted [9].

\subsection{Model and Analysis of Ship Radiated Noise}

The low-frequency line spectrum component is associated with the propulsion system, propeller and auxiliary equipment. The line spectrum quantity produced by auxiliary equipment is generally quite stable, and it is independent of the ship speed [10]. Moreover, the bandwidth of this line spectrum is generally proportional to the frequency, and its scope is $0.03 \%-0.3 \%$ of the center frequency. In view of the above-mentioned characteristics, this paper adopts the model of four-blade propeller ship as the experimental object, whose shaft frequency is $f_{0}=5 \mathrm{~Hz}$. Then, the model of ship radiated noise is established as follows:

$$
\left\{\begin{array}{l}
s_{1}(\mathrm{t})=4.9 \cos \left(2 \pi f_{0} \mathrm{t}\right) \\
s_{2}(\mathrm{t})=2.9 \cos \left(4 \pi f_{0} \mathrm{t}-7.1\right) \\
s_{3}(\mathrm{t})=1.5 \cos \left(6 \pi f_{0} \mathrm{t}-9\right) \\
s_{4}(\mathrm{t})=0.9 \cos \left(8 \pi f_{0} \mathrm{t}+2\right) \\
s_{5}(\mathrm{t})=15 n(\mathrm{t})
\end{array}\right.
$$

where, $n(\mathrm{t})$ denotes Gauss white noise. As can be seen from the model, low-frequency line spectrum contains four frequency components and stronger random noise. The four frequencies are $5 \mathrm{~Hz}, 10 \mathrm{~Hz}, 15 \mathrm{~Hz}$ and $20 \mathrm{~Hz}$ respectively, and each frequency component is larger than $1 \mathrm{~Hz}$. At this time, the conventional stochastic resonance cannot meet the requirements any more. Meanwhile, the time scale transformation method for large parameters has the shortcoming of over-large errors [11].

\subsection{System Calculation}

In the simulation experiment, we adopted four-order Runge-Kutta algorithm to implement the numerical solution of system, and the specific calculation process is shown as follows:

$$
\left\{\begin{array}{l}
k 1=h *\left[\mathrm{a}^{*} y(\mathrm{n})-\mathrm{b}^{*} y(\mathrm{n})^{3}+x(\mathrm{n})\right] \\
k 2=h *\left[\mathrm{a}^{*}(y(\mathrm{n})+k 1 / 2)-\mathrm{b}^{*}(y(\mathrm{n})+k 1 / 2)^{3}+x(\mathrm{n})\right] \\
k 3=h *\left[\mathrm{a}^{*}(y(\mathrm{n})+k 2 / 2)-\mathrm{b}^{*}(y(\mathrm{n})+k 2 / 2)^{3}+x(\mathrm{n}+1)\right] \\
k 4=h *\left[\mathrm{a}^{*}(y(\mathrm{n})+k 3)-\mathrm{b}^{*}(y(\mathrm{n})+k 3)^{3}+x(\mathrm{n}+1)\right] \\
y(\mathrm{n}+1)=y(\mathrm{n})+\frac{1}{6} *(k 1+2 * k 2+2 * k 3+k 4)
\end{array}\right.
$$

where, $y(n)$ is the output signal with the initial value $y(0)=0, x(\mathrm{n})$ is the input signal, and $h$ is the calculation step, i.e., the reciprocal of the sampling frequency.

\subsection{Sweep-Frequency Stochastic Resonance}

According to the as-built model of ship radiated noise, we can see that the low-frequency line spectrum contains the large parameter signals totally. In order to detect such signals, we will employ the sweep frequency stochastic resonance. The measured signal is shown as follows:

$$
S(\mathrm{t})=\sum_{i=1}^{4} A_{i} \cos \left(2 \pi f_{i} \mathrm{t}+\varphi_{i}\right)+15 n(\mathrm{t})
$$

Then, we add a frequency sweeping carrier signal to modulate the measured signal, where the frequency sweeping carrier signal is given as follows.

$$
Y(\mathrm{t})=\cos \left(2 \pi f_{c} \mathrm{t}\right)
$$

At this time, the modulation signal output is obtained as follows. 


$$
\begin{aligned}
& S(\mathrm{t}) \times \mathrm{Y}(\mathrm{t}) \\
= & {\left[\sum_{i=1}^{4} A_{i} \cos \left(2 \pi f_{i} \mathrm{t}+\varphi_{i}\right)+15 n(\mathrm{t})\right] \times \cos \left(2 \pi f_{c} \mathrm{t}\right) } \\
= & \frac{1}{2} \sum_{i=1}^{4} A_{i} \cos \left[2 \pi\left(f_{i}+f_{c}\right) \mathrm{t}+\varphi_{i}\right]+ \\
& \frac{1}{2} \sum_{i=1}^{4} A_{i} \cos \left[2 \pi\left(f_{i}-f_{c}\right) \mathrm{t}+\varphi_{i}\right]+15 n(\mathrm{t}) \cos \left(2 \pi f_{c} \mathrm{t}\right)
\end{aligned}
$$

The above equation is composed of three parts. The first part is $X_{1}=\frac{1}{2} \sum_{i=1}^{4} A_{i} \cos \left[2 \pi\left(f_{i}+f_{c}\right) \mathrm{t}+\varphi_{i}\right]$, which is the sum value of the frequencies of measured signal and sweepfrequency carrier signal. Moreover, with regard to this part of signal, its frequency is far more than the applicable scope of stochastic resonance. Therefore we will use the low-pass filter to remove this part of signal in the next section. The second part is $X_{2}=\frac{1}{2} \sum_{i=1}^{4} A_{i} \cos \left[2 \pi\left(f_{i}-f_{c}\right) \mathrm{t}+\varphi_{i}\right]$, which is the difference value of the frequencies of measured signal and sweep-frequency signal. We intend to constantly approximate the signal frequency $f_{i}$ to be measured through adjusting the sweeping frequency $f_{c}$. In the approximation process, if the difference value is small enough to satisfy the adiabatic approximation theory, the system will produce the effect of stochastic resonance, i.e., the output signal spectrum corresponding to each $f_{c}$ will exist a obvious non- zero peak. Especially when there is $f_{c}=f_{i}$, the effect of stochastic resonance does not exist, and the corresponding maximum power spectrum peak is close to 0 , i.e., its reciprocal is infinite. Therefore, we can use this feature to extract the frequency components contained in the lowfrequency line spectrum. The third part is the modulation noise $X_{3}=15 n(\mathrm{t}) \cos \left(2 \pi f_{c} \mathrm{t}\right)$, and we can find that it still belongs to Gauss white noise through obtaining its autocorrelation function and power spectrum [12]. In the process of adjusting the sweep-frequency carrier, we make partitioning and adaptive selection on system noise strength according to the SNR gain. Moreover, we estimate the power spectrum of the output signal of stochastic resonance system, and extract the reciprocal of the corresponding spectral peak, whose curve will be drawn along with the change of frequency sweeping carrier. In this curve, each sharp peak corresponds to the frequency components of low-frequency line spectrum. The block diagram of the concrete system is illustrated in Fig. (1).

\section{SIMULATION ANALYSIS}

\subsection{The Detection of Single-Frequency Weak Signal}

We assume a sinusoidal signal, whose magnitude is $A=0.3$ and frequency $f=0.01 \mathrm{~Hz}$. The system parameters are $a=0.7434$ and $b=0.1$, the noise intensity is $D=4$, and the sampling frequency is $f_{s}=5 \mathrm{~Hz}$. As seen from Fig.

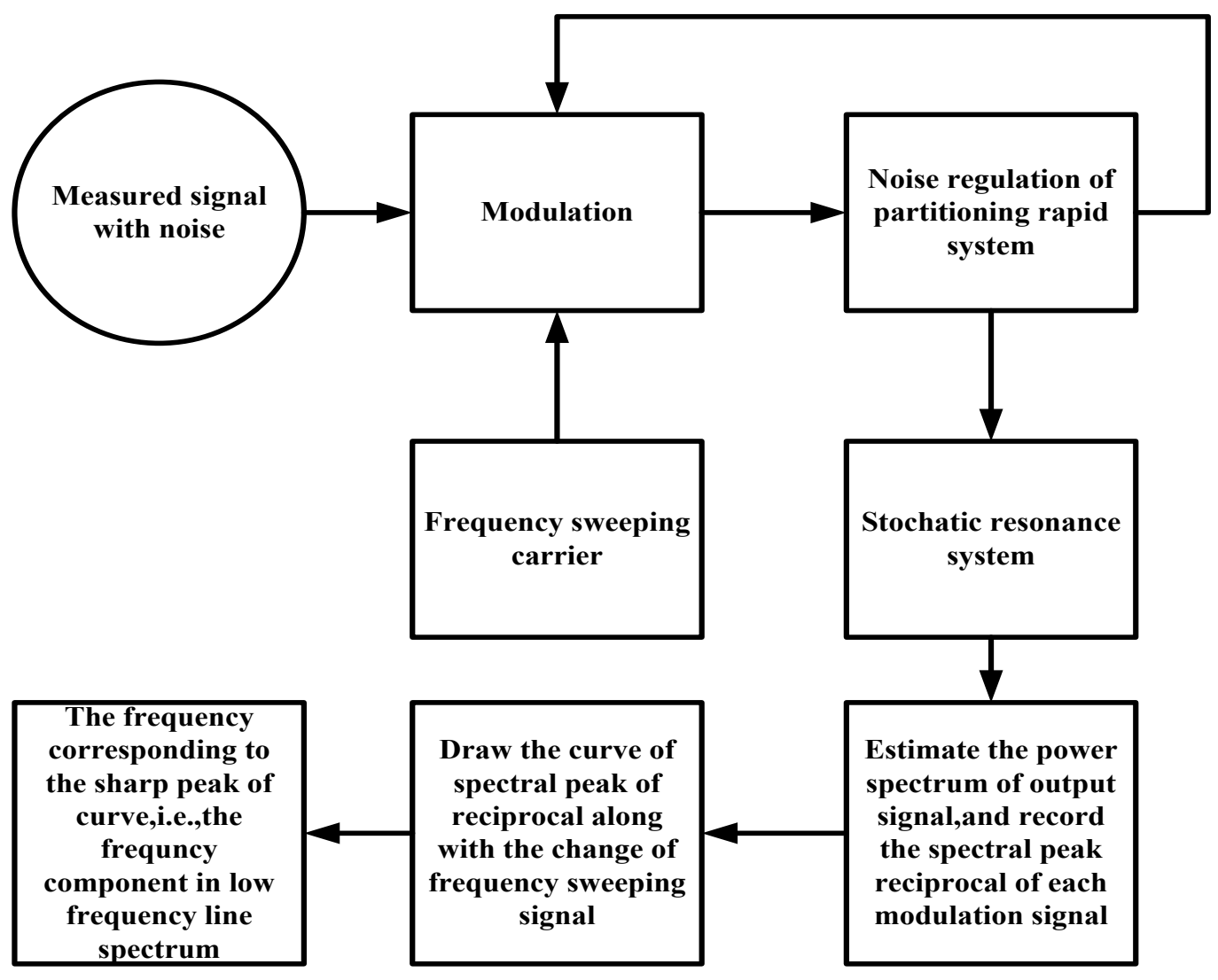

Fig. (1). The block diagram of sweep frequency stochastic resonance system. 
(a) Input signal

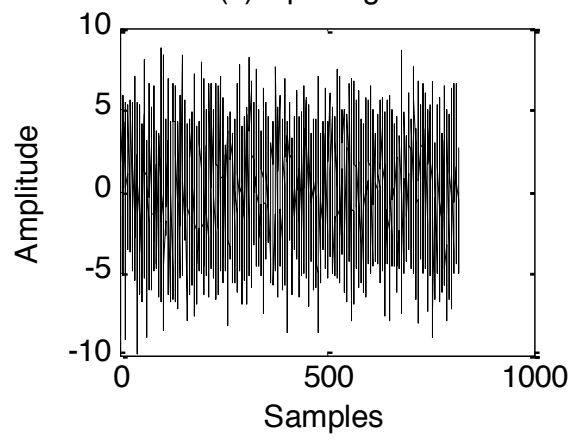

(c) Output signal

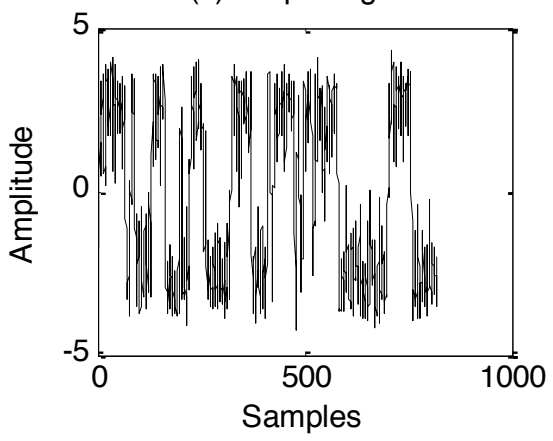

(b) Spectrum of input signal

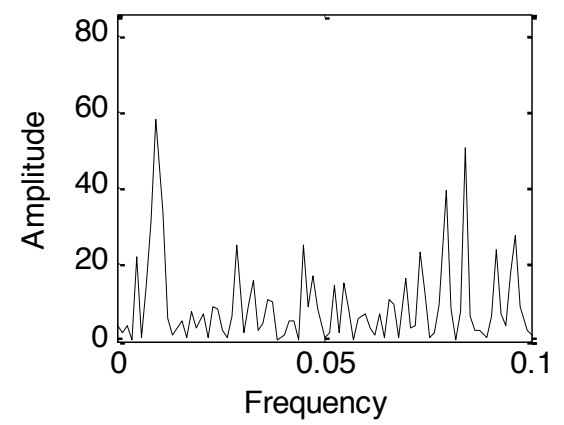

(d) Spectrum of output signal

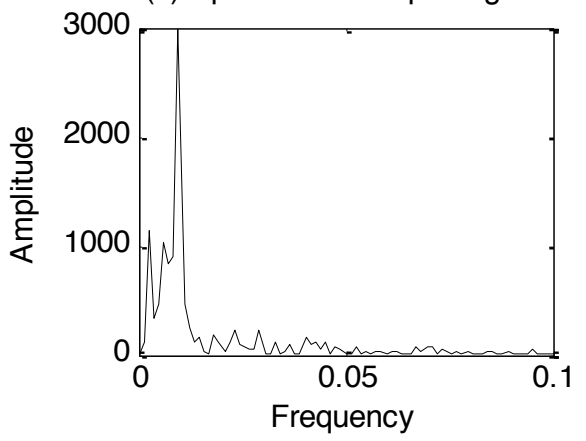

Fig. (2). Single-frequency stochastic resonance.

(2a, b), the general spectral method cannot get the measured signal when the noise intensity reaches to 4 . Fig. (2d) shows the frequency spectrum of system output, and we can find that there is an apparent sharp spectral peak at $f=0.01 \mathrm{~Hz}$, which greatly improves the output SNR. Moreover, Fig. (2c) shows the time-domain characteristic of output signal, and we can find that the output obviously presents the periodicity as the input sinusoidal signal after being processed by the stochastic resonance system. We set the constant parameters $b=0.1$ and $D=4$, and regulate the parameter $a$ from 0.1 to 5 . Then, we can clearly see the phenomenon of stochastic resonance which is resulted from parameter regulation, just as shown in Fig. (2). When the parameter $a$ is gradually increased, the output SNR monotonously increases in first, and then decreases monotonically. When there exists $a=0.7434$, the best state of stochastic resonance is achieved. At this time, the SNR gain is $S N R_{\text {gain }}=142.3$

\subsection{The Detection of Multi-Frequency Weak Signal}

In view of the above model, we set the sampling frequency $f_{s}=100 \mathrm{~Hz}, \mathrm{~b}=1$, and the remainder parameters are unchanged. Then, the relationship curve between SNR gain and parameter is shown as in Fig. (3).

As seen in Fig. (4), the SNR gain of multi-frequency signal presents a disorganized change along with the change of system parameter a, which reflects that the stochastic resonance system did not play an effective role. We take the value of peak in Fig. (4) as the system parameter value, i.e., $\mathrm{a}=1.733$, and then observe the time/frequency domain characteristics of input and output signals, which are shown

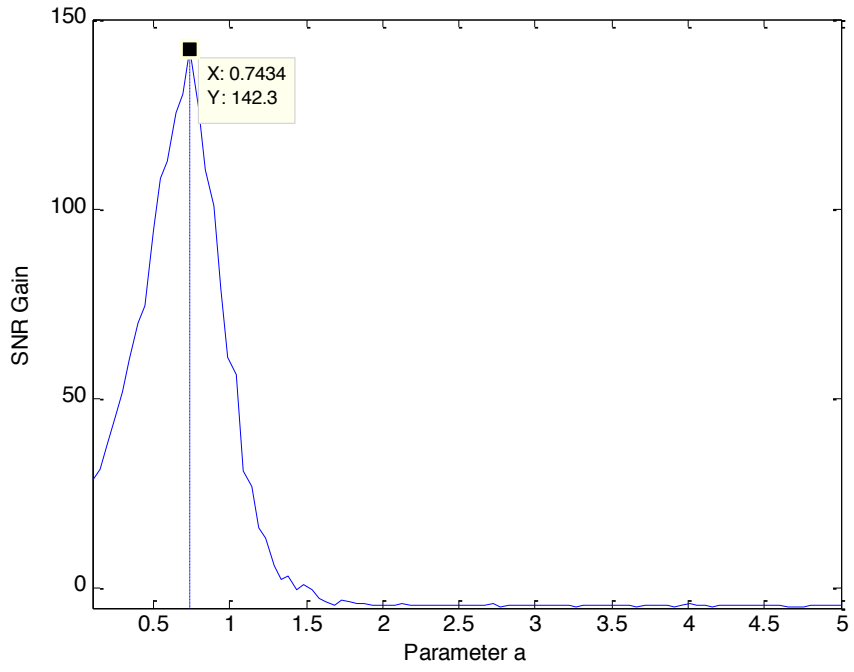

Fig. (3). The SNR gain of single-frequency weak signal along with the change of system parameter a.

in Fig. (5). As seen in Fig. (5b), we can find that lowfrequency line spectrum component is completely submerged in the background noise. Meanwhile, Fig. (5d) presents that line spectrum component is completely not recognized from the spectrum after the treatment of stochastic resonance system. Moreover, we can also see that the high-frequency noise transfers to the low-frequency band, which verifies that the stochastic resonance system totally losses its effectiveness at this time.

\subsection{The Detection of Sweep Frequency Stochastic Resonance}

The system model is shown as Eq. (4), and we can find that the radiated noise model includes four frequency 
components, where the amplitude and the phase of each frequency component are not the same. It is difficult for us to find an optimal system parameter or noise strength just to meet all the frequency components. Based on the characteristics that the shaft-frequency signal has base frequency, and the rest line spectrum is the low-frequency line spectrum of doubling shaft frequency, we first estimate the range of shaft frequency, and then adaptively adjust the noise intensity of partitioning system according to this range. According to the model, we first estimate the range from 4 $\mathrm{Hz}$ to $6 \mathrm{~Hz}$, and then orderly divide three regions $[6 \mathrm{~Hz}, 12 \mathrm{~Hz}],[12 \mathrm{~Hz}, 16 \mathrm{~Hz}]$ and $[16 \mathrm{~Hz}, 21 \mathrm{~Hz}]$. We solve the optimal system noise intensity for each region, where the system parameters are $a=7.2$ and $b=1$, the sampling frequency is $f_{s}=5 \mathrm{kHz}$, the interval frequency is $f_{c}=0.02$ $\mathrm{Hz}$, the range of noise intensity is $[0.1,50]$, and the step size is 0.1 . Then, the simulation results are shown in Fig. (6).

As seen in Fig. (6), there appear obvious sharp peaks at multiple frequencies $5 \mathrm{~Hz}, 10 \mathrm{~Hz}, 15 \mathrm{~Hz}$ and $20 \mathrm{~Hz}$. Then, the low-frequency line spectrum submerging in strong noise is detected, and the effectiveness of the proposed method is also verified. Furthermore, after the fast regulation, the corresponding noise intensities in different partitions are 37 , 26,18 and 0.8 , respectively.

\section{CONCLUSION}

In this paper, starting from the stochastic resonance system, we firstly carried out the theoretical analysis on stochastic resonance, and described its applicable scope. Subsequently, we established the radiated noise model of four-blade propeller ship and analyzed its characteristics. Based on these characteristics, we proposed a sweepfrequency stochastic resonance detection system, which can

(a) Input signal

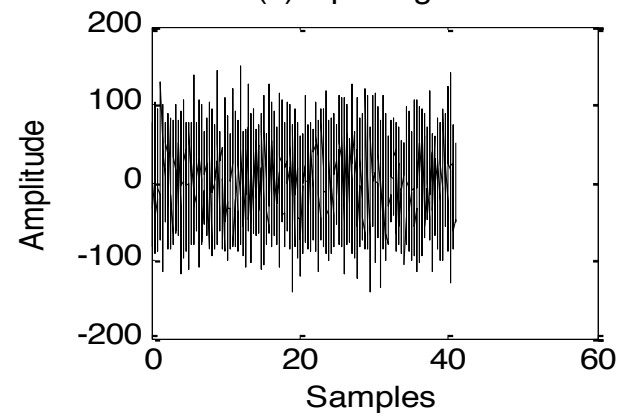

(c) Output signal

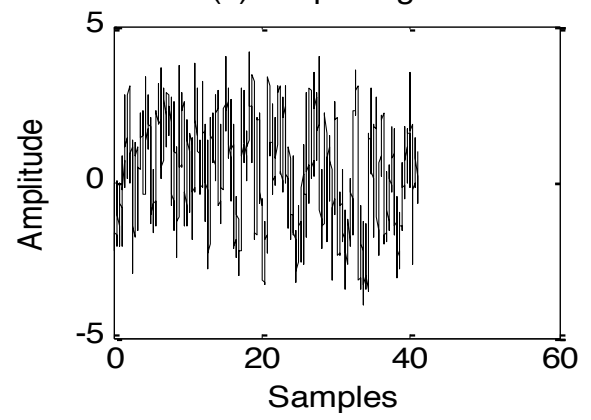

quickly select the system noise for corresponding partition, according to the SNR gain. Simulation results present that, the proposed system can detect the multi-frequency signal with large parameters quickly and accurately. Under the strong noise background, it can still successfully detect the low-frequency line spectrum component in ship radiated noise. Therefore, it has great application value and development prospect, meanwhile it lays the solid foundation for the subsequent target recognition and speed estimation of the ship.

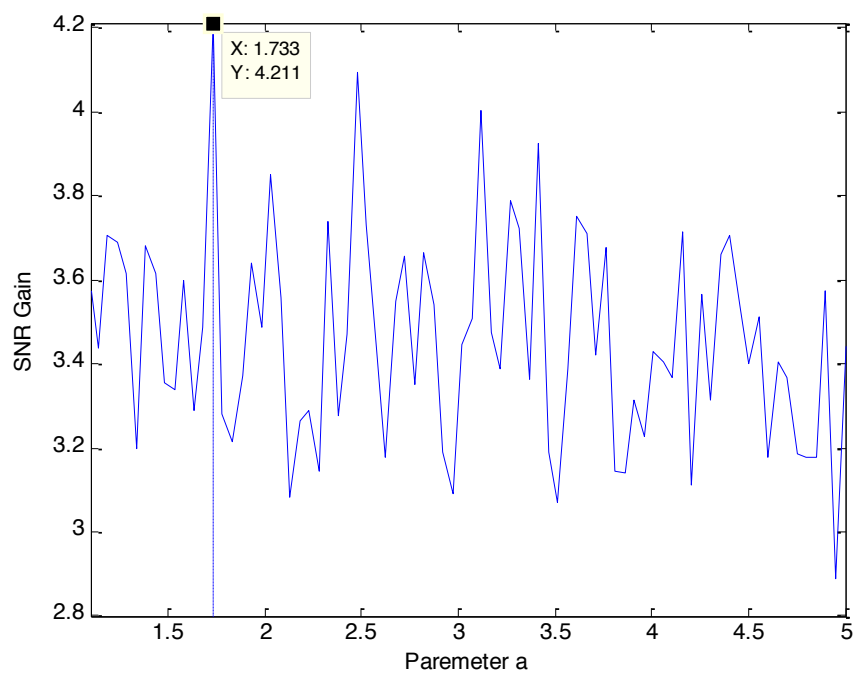

Fig. (4). The SNR gain of multi-frequency weak signal along with the change of system parameter a.

\section{CONFLICT OF INTEREST}

The authors confirm that this article content has no conflict of interest.

(b) Spectrum of input signal

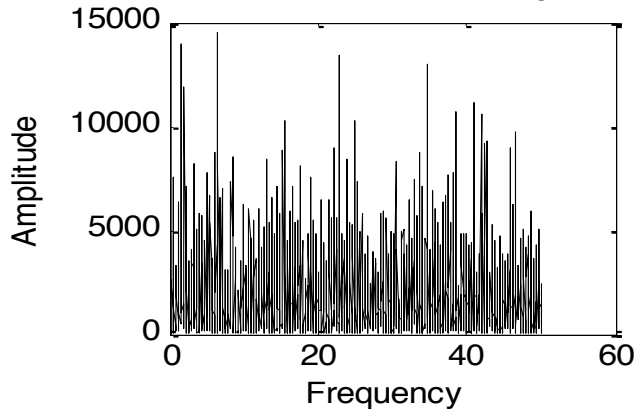

(d) Spectrum of output signal

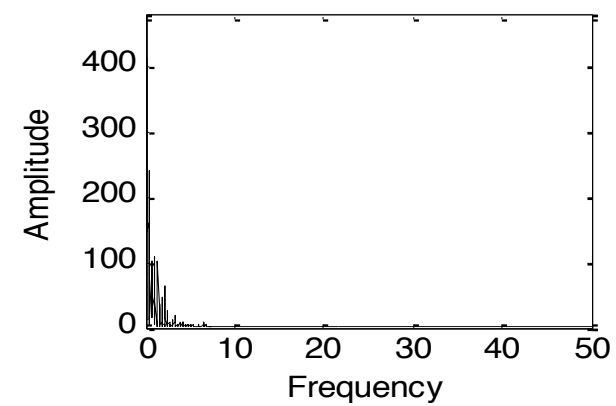

Fig. (5). Stochastic resonance of multi-frequency signal with large parameters. 


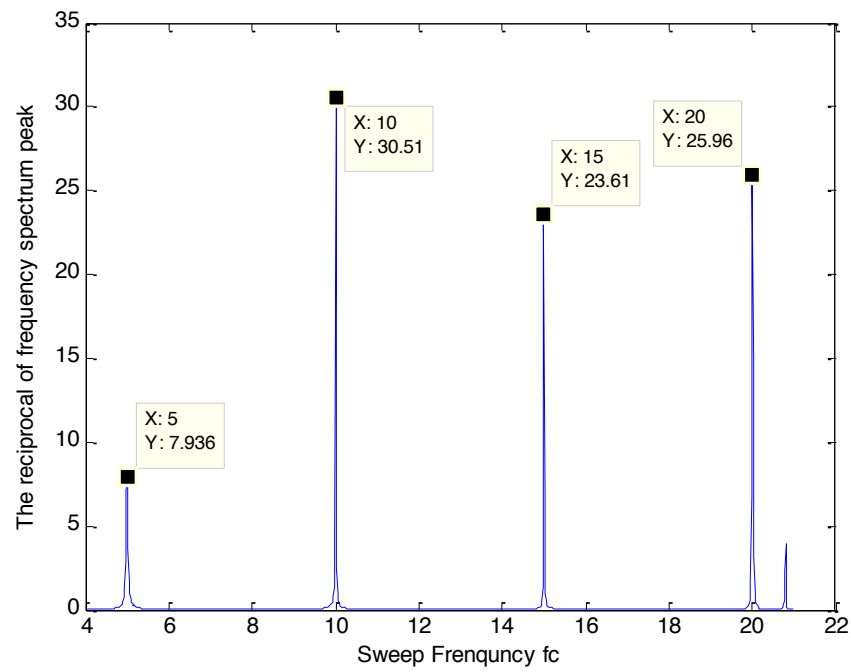

Fig. (6). The curve of spectral peak reciprocal along with the change of sweeping frequency.

\section{ACKNOWLEDGEMENTS}

This work was supported by the National Natural Science Foundation of China (61471309, 61107023), Ph.D. Programs Foundation of Ministry of Education of China (20110121120020), the Fujian Natural Science Foundation of China (2013J01258), and the key project in Fujian Province (2012H 1012).

\section{REFERENCES}

[1] S. Ianniello, R. Muscari, M. A. Di, "Ship underwater noise assessment by the acoustic analogy, part III: measurements versus numerical predictions on a full-scale ship," Journal of Marine Science and Technology, vol. 19, no.2, pp.125-42, 2014.
[2] S.E. Crocker, P.L. Nielsen, J.H. Miller, and M. Siderius, "Geoacoustic inversion of ship radiated noise in shallow water using data from a single hydrophone," Journal of the Acoustical Society of America, vol. 136, no. 5, pp. EL362-EL8, 2014.

[3] G.Z. Shi, and J.C. Hu, "Theoretical analysis of the ship noise demodulation line spectrum structure," Harbin Gongcheng Daxue Xuebao/Journal of Harbin Engineering University, vol. 28, pp. 138-42, 2007.

[4] R. Benzi, "Stochastic resonance: from climate to biology," Nonlinear Processes in Geophysics, vol.17, no.5, pp.431-41, 2010.

[5] Z. Lu, J. Lin, G. Hu, "Numerical study of Fokker-Planck equation for stochastic resonance problem," Wuli Xuebao/Acta Physica Sinica, vol. 42, no. 10, pp. 1556-66, 1993.

[6] M. Lin, and Y.M. Huang, "The mechanism of energy input to bistable system to generate stochastic resonance," Acta Physica Sinica, vol. 61, no.22, 2012.

[7] H.Q. Zhang, W. Xu, C.Y. Sun, and Y. Xu, "Stochastic resonance in a bistable system driven by weak periodic signal with multiple delays," International Journal of Modern Physics B, vol. 25, no.13, pp. 1775-83, 2011.

[8] J. Wang, Q. He, and F. Kong, "An improved multiscale noise tuning of stochastic resonance for identifying multiple transient faults in rolling element bearings," Journal of Sound and Vibration, vol. 333, no.26, pp.7401-7421, 2014.

[9] Y. Qin, Y. Tao, Y. He, and B. Tang, "Adaptive bistable stochastic resonance and its application in mechanical fault feature extraction," Journal of Sound and Vibration, vol. 333, no. 26, pp. 7386-7400, 2014.

[10] K. Listewnik, I. Gloza, "Underwater ship noise spatial distribution of chosen main mechanisms in shallow water," $17^{\text {th }}$ International Congress on Sound and Vibration 2010, ICSV 2010, July 18, 2010 - July 22, 2010, Cairo, Egypt: International Institute of Acoustics and Vibrations, 2010.

[11] G.F. Wang, H.R. Zhang, F.Q. Zhang, J.C. Ye, and L. Wei, "Scale transformation for detecting weak periodic signal of stochastic resonance," 2010 IEEE International Conference on Intelligent Computing and Integrated Systems, ICISS2010, October 22, 2010 October 24, 2010; Guilin, China: IEEE Computer Society, 2010.

[12] K. Hayashi, S. de Lorenzo, M. Manosas, J.M. Huguet, F. Ritort, "Single-Molecule Stochastic Resonance," Physical Review X, vol. 2, no.3, 2012.

This is an open access article licensed under the terms of the (https://creativecommons.org/licenses/by/4.0/legalcode), which permits unrestricted, non-commercial use, distribution and reproduction in any medium, provided the work is properly cited. 